\title{
An Annotation Scheme for Free Word Order Languages
}

\author{
Wojciech Skut, Brigitte Krenn, Thorsten Brants, Hans Uszkoreit \\ Universität des Saarlandes \\ 66041 Saarbrücken, Germany \\ \{skut, krenn, brants, uszkoreit\}@coli.uni-sb.de
}

\begin{abstract}
We describe an annotation scheme and a tool developed for creating linguistically annotated corpora for non-configurational languages. Since the requirements for such a formalism differ from those posited for configurational languages, several features have been added, influencing the architecture of the scheme. The resulting scheme reflects a stratificational notion of language, and makes only minimal assumptions about the interrelation of the particular representational strata
\end{abstract}

\section{Introduction}

The work reported in this paper aims at providing syntactically annotated corpora ('treebanks') for stochastic grammar induction. In particular, we focus on several methodological issues concerning the annotation of non-configurational languages.

In section 2, we examine the appropriateness of existing annotation schemes. On the basis of these considerations, we formulate several additional requirements. A formalism complying with these requirements is described in section 3. Section 4 deals with the treatment of selected phenomena. For a description of the annotation tool see section 5 .

\section{Motivation}

\subsection{Linguistically Interpreted Corpora}

Combining raw language data with linguistic information offers a promising basis for the development of new efficient and robust NLP methods. Realworld texts annotated with different stratia of linguistic information can be used for grammar induction. The data-drivemness of this approach presents a clear advantage over the traditional, idealised notion of competence grammar.

\subsection{Existing Treebank Formats}

Corpora annotated with syutactic structures are commonly referred to as tretbankis. Existing tree- bank annotation schemes exhibit a fairly uniform architecture, as they all have to meet the same basic requirements, namely:

Descriptivity: Grammatical phenomena are to be described rather than explained.

Theory-independence: Annotations should not be influenced by theory-specific considerations. Nevertheless, different theory-specific representations shall be recoverable from the annotation, cf. (Marcus et al., 1994).

Multi-stratal representation: Clear separation of different description levels is desirable.

Data-drivenness: The scherne must provide representational means for all phenomena occurring in texts. Disambiguation is based on human processing skills (cf. (Marcus et al., 1994), (Sampson, 1995), (Black et. al. , 1996)).

The typical treebank architecture is as follows:

Structures: A context-free backbone is augmented with trace-filler representations of non-local dependencies. The underlying argument structure is not represented directly, but can be recovered from the tree and trace-filler ammotations.

Syntactic category is encoded in node labels.

Grammatical functions constitute a complex label system (cf. (Bies et al., 1995), (Sampson, 1995)).

Part-of-Speech is annotated at word level.

Thus the context-free constituent backbone plays a pivotal role in the annotation scheme. Due to the substantial differences between existing models of constitnent structure, the question arises of how the theory independence requirement can be satisfied. At this point the importance of the underlying argument structure is emphasised (cf. (Lehmann et al., 1996), (Marcus et al., 1994), (Sampson, 1995)).

\subsection{Language-Specific Features}

Treebanks of the format described in the above section have been designed for English. Therefore, the 
solutions they offer are not always optimal for other language types. As for free word order languages, the following features may cause problems:

- local and non-local dependencies form a contimumin rather than clear-cut classes of phenomena;

- there exists a rich inventory of discontinuous constituency types (topicalisation, scrambling, clause union, pied piping, extraposition, split NPs and PPs);

- word order variation is sensitive to many factors, e.g. category, syntactic function, focus;

- the grammaticality of different word permutations does not fit the traditional binary 'rightwrong" pattern; it rather forms a gradual transition between the two poles.

In light of these facts, serious difficulties can be expected arising from the structural component of the existing formalisms. Due to the frecuency of discontinuous constituents in non-configurational languages, the filler-trace mechanism would be used very often, yielding syntactic trees fairly different from the underlying predicate-argument structures.

Consider the German sentence

(1) daran wird ihn Anna erkennen, daß er weint at-it will him Anua recognise that he cries 'Anna will recognise him at his cry'

A sample constituent structure is given below:

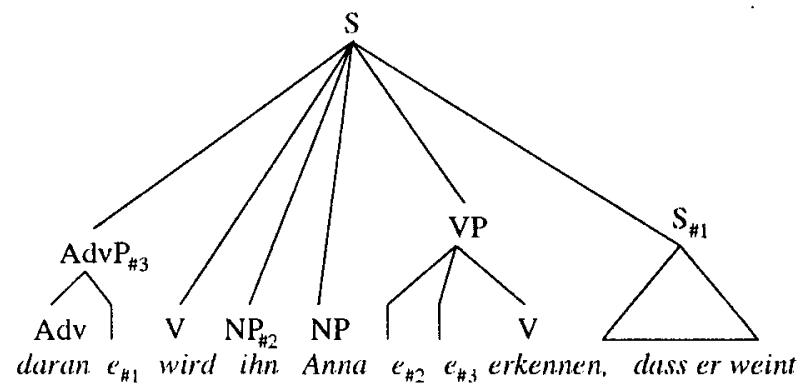

The fairly short sentence contains three non-local dependencies, marked by co-references between traces and the corresponding nodes. This hybrid representation makes the structure less transparent, and therefore more difficult to annotate.

Apart from this rather technical problem, two further arguments speak against phrase structure as the structural pivot of the annotation scheme:

- Phrase structure models stipulated for nonconfigurational languages differ strongly from each other, presenting a challenge to the intended theory-independence of the scheme.

- Constituent structure serves as an explanatiory device for word order variation, which is difficult to reconcile with the descriptivity requirement.
Finally, the structural handling of free word order means stating well-formedness constraints on structures involving many trace-filler dependencies, which has proved tedious. Since most methods of handling discontinuous constituents make the formalism more powerful, the efficiency of processing deteriorates, too.

An alternative solution is to make argument structure the main structural component of the formalism. This assumption underlies a growing number of recent syntactic theories which give up the context-free constituent backbone, cf. (McCawley, 1987), (Dowty, 1989), (Reape, 1993), (Kathol and Pollard, 1995). These approaches provide an adequate explanation for several issues problematic for phrase-structure grammars (clause union, extraposition, diverse second-position phenomena).

\subsection{Annotating Argunent Structure}

Argument structure can be represented in terms of unordered trees (with crossing branches). In order to reduce their ambiguity potential, rather simple, 'flat.' trees should be employed, while more information can be expressed by a rich system of function labels.

Furthermore, the required theory-independence means that the form of syntactic trees should not reflect theory-specific assumptions, e.g. every syntactic structure has a micpue head. Thus, notions such as head should be distinguished at the level of syntactic functions rather than structures. This requirement speaks against the traditional sort of $d t$ : pendency trees, in which heads are represented as non-terminal nodes, cf. (Hudson, 1984).

A tree meeting these requirements is given below:

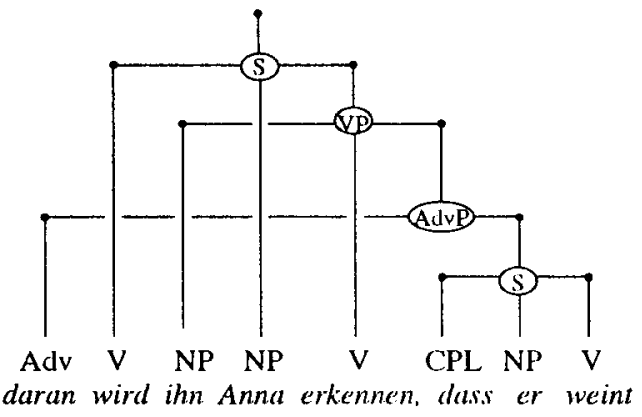

Such a word order independent representation has the advantage of all structural information being encoded in a single data structure. A uniform representation of local and non-local dependencies makes the structure more transparent ${ }^{1}$.

\section{The Annotation Scheme}

\subsection{Architecture}

We distinguish the following levels of representation:

\footnotetext{
${ }^{1} \mathrm{~A}$ context-free constituent backbone can still be recovered from the surface string and argument structure by reattaching 'extracted' structures to a higher node.
} 
Argument structure, represented in terms of unordered trees.

Grammatical functions, encoded in edge labels, e.g. SB (subject), MO (modifier), HD (head).

Syntactic categories, expressed by category labels assigned to non-terminal nodes and by part-of-speech tags assigned to terminals.

\subsection{Argument Structure}

A structure for (2) is shown in fig. 2 .

(2) schade, dał kein Arzt anwesend ist, der pity that no doctor present is who sich anskennt

is competent

'Pity that no competent doctor is here'

Note that the root node does not have a head descendant (HD) as the sentence is a predicative construction consisting of a subject (SB) and a predicate (PD) without a copula. The subject is itself a sentence in which the copula (ist) does occur and is assigned the tag $\mathrm{HD}^{2}$.

The tree resembles traditional constituent, structures. The difference is its word order independence: structural units ("phrases") need not be contiguous substrings. For instance, the extraposed relative clause (RC) is still treated as part of the subject NP.

As the annotation scheme does not distinguish different bar levels or any similar intermediate categories, only a small set of node labels is needed (currently 16 tags, S, NP, AP ...).

\subsection{Grammatical Functions}

Due to the rudimentary character of the argument structure representations, a great deal of information has to be expressed by grammatical functions. Their further classification must reflect different kinds of linguistic information: morphology (e.g., case, inflection), category, dependency type (complementation vs. modification), thematic role, etc. ${ }^{3}$

However, there is a trade-off between the granularity of information encoded in the labels and the speed and accuracy of annotation. In order to avoid inconsistencies, the corpus is annotated in two stages: basic annotation and refinement. While in the first phase each annotator has to annotate structures as well as categrories and functions, the refinement. can be done separately for each representation level.

During the first phase, the focus is on annotating correct structures and a coarse-grained classification of grammatical functions, which represent the following areas of information:

\footnotetext{
${ }^{2} \mathrm{CP}$ stands for complementizer, $\mathrm{OA}$ for accusative object and RC for relative clause. NK denotes a kernel NP' component ( $\mathrm{v}$. section 4.1).

${ }^{3}$ For an extensive use of grammatical functions of. (Karlsson et al., 1995), (Voutilainen, 1994).
}

Dependency type: complemcuts are further classified according to features such as category and case: clausal complements (OC), accusative objects (OA), datives (DA), etc. Modifiers are assigned the label MO (further classification with respect to thematic roles is planned). Separate labels are defined for dependencies that do not fit. the complement/modifier dichotomy, e.g., pre- (GL) and postnominal genitives (GR).

Headedness versus non-headedness:

Headed and non-headed structures are distinguished by the presence or alssence of a branch labeled HD.

Morphological information: Another set of labels represents morphological information. PM stands for morphological particle, a label for German infinitival $z u$ and superlative am. Separable verb prefixes are labeled SVP.

During the second annotation stage, the annotation is enriched with information about thematic roles, quantifier scope and anaphoric reference. As already mentioned, this is done separately for each of the three information areas.

\subsection{Structure Sharing}

A phrase or a lexical item can perform multiple functions in a sentence. Consider tqui ucrls where the subject of the infinitival VP is not realised syntactically, but co-referent with the subject or object of the matrix equi verb:

(3) er bat mich zu kommen he asked me to colne

(mich is the understood subject of kommen.). In such cases, an additional edge is drawn from the embedded VP node to the controller, thus changing the syntactic tree into a graph. We call such additional edges secondary links and represent them as dotted lines, see fig. 4, showing the structure of (3).

\section{Treatment of Selected Phenomena}

As theory-independence is one of our objectives, the annotation scheme incorporates a number of widely accepted linguistic analyses, especially in the area of verbal, adverbial and adjectival syntax. However, some other standard analyse.s turn ont to be problematic, mainly due to the partial, idealised character of competence grammars, which often marginalise or ignore such important phenomena as 'deficient' (e.g. headless) constructions, appositions, temporal expressions, etc.

In the following paragraphs, we give annotations for a number of such phenomena.

\subsection{Noun Phrases}

Most linguistic theories treat. NPs as structures headed by a unique lexical item (nom). However, this 
idealised model needs several additional assumptions in order to account for such inuportant phenomena as complex nominal NP components (cf. (4)) or nominalised adjectives ( $\mathrm{f}$. (5)).

(4) my uncle Peter Snith

(5) der sehr Glïckliche

the very happy

'the very happy one'

In (4), different theories make different headedness predictions. In (5), either a lexical nominalisation rule for the adjective Glückliche is stipulated, or the existence of an empty nominal head. Moreover, the so-called DP analysis views the article $d e r$ as the head of the phrase. Further differences concern the attachment of the degree modifier sehr.

Because of the intended theory-independence of the scheme, we annotate only the common minimum. We distinguish an NP kernel consisting of determiners, adjective phrases and nomms. All components of this kermel are assigned the label NK and treated as sibling nodes.

The difference between the particular NK's lies in the positional and part-of-speech information, which is also sufficient to recover theory-specific structures from our 'underspecified' representations. For instance, the first determiner among the NK's can be treated as the specifier of the phrase. The head of the phrase can be determined in a similar way according to theory-specific assumptions.

In addition, a number of clear-cut NP components can be defined outside that juxtapositional kernel: pre- and postmominal genitives (GL, GR), relative clauses (RC), clausal and sentential complements (OC). They are all treated as siblings of NK's regardless of their position (in situ or extraposed).

\subsection{Attachment Ambiguities}

Adjunct attachment often gives rise to structural ambiguities or structural uncertainty. However, full or partial disambiguation takes place in context, and the annotators do not consider unrealistic readings.

In addition, we have adopted a simple convention for those cases in which context information is insufficient for total disambignation: the highest possible attachment site is chosen.

A similar convention has been adopted for constructions in which scope ambiguities have syutactic effects but a one-to-one correspondence between scope and attachment does not seem reasonable, of. focus particles such as only or also. If the scope of such a word does not directly correspond to a tree node, the word is attached to the lowest node dominating all subconstituents appearing in its scope.

\subsection{Coordination}

A problem for the rudimentary argument structure representations is the use of incomplete structures in natural language, i.e. phenomena such as coordination and ellipsis. Since a precise structural description of non-constituent coordination would require a rich inventory of incomplete phrase types, we have agreed on a sort of underspecified representations: the coordinated units are assigned structures in which missing lexical material is not represented at the level of primary links. Fig. 3 shows the representation of the sentence:

(6) sie wurde von preubischen Truppen besetzt she was by Prussian troops occupied und 1887 dem preubischen Strat. angegliedert and 1887 to-the Prussian state incorporated 'it was occupied by Prussian troops and incorporated into Prussia in $1887^{\prime}$

The category of the coordination is labeled CVP here, where $C$ stands for coordination, and VP for the actual category. This extra marking makes it easy to distinguish between 'normal' and coordinat.ed categories.

Multiple coordination as well as enumerations are amnotated in the same way. An explicit coordinating conjunction need not be present.

Structure-sharing is expressed using secondary links.

\section{The Annotation Tool}

\subsection{Requirements}

The development of linguistically interpreted corpora presents a laborious and time-consuming task. In order to make the annotation process more efficient, extra effort has been put into the development, of an annotation tool.

The tool supports immediate graphical feedback and automatic error checking. Since our scheme permits crossing edges, visualisation as bracketing and indentation would be insufficient. Instead, the complete structure should be represented

The tool should also permit a convenient handling of node and edge labels. In particular, variable tagsets and label collections should be allowed.

\subsection{Implementation}

As the need for certain functionalities becomes obvious with growing annotation experience, we have decided to implement the tool in two stages. In the first phase, the main functionality for building and displaying unordered trees is supplied. In the second phase, secondary links and additional structural functions are supported. The implementation of the first phase as described in the following paragraphs is completed.

As keyboard input is more efficient than mouse input (cf. (Lehmann et al., 1996)) most effort has been put in developing an efficient keyboard interface. Menus are supported as a useful way of getting 
help on commands and labels. In addition to pure annotation, we can attach comments to structures.

Figure 1 shows a screen dump of the tool. The largest part of the window contains the graphical representation of the structure being amotated. The following commands are available:

- group words and/or phrases to a new phrase;

- ungroup a phrase;

- change the name of a phrase or an edge;

- re-attach a node;

- generate the postscript output of a sentence.

The three tagsets used by the annotation tool (for words, phrases, and edges) are variable and are stored together with the corpus. This allows easy modification if needed. The tool checks the appropriateness of the input.

For the implementation, we used Tcl/Tk Version 4.1. The corpus is stored in a SQL database.

\subsection{Automation}

The degree of automation increases with the amount. of data available. Sentences annotated in previous steps are used as training material for further processing. We distinguish five degrees of automation:

0) Completely manual annotation.

1) The user deternines phrase boundaries and syntactic categories ( $S, N P$, etc.). The program automatically assigns grammatical function labels. The annotator can alter the assigned tags.

2) The user only determines the components of a new phrase, the program determines its syntactic category and the grammatical functions of its elements. Again, the annotator has the option of altering the assigned tags.

3) Additionally, the program performs simple bracketing, i.e., finds 'kernel' phrases.

4) The tagger suggests partial or complete parses.

So far, about 1100 sentences of our corpus have been annotated. This amount of data suffices as training material to reliably assign the grammatical functions if the user determines the elements of a phrase and its type (step 1 of the list, above).

\subsection{Assigning Grammatical Function Labels}

Grammatical functions are assigned using standard statistical part-of-speech tagging methods (cf. e.g. (Cutting et al., 1992) and (Feldweg, 1995))

For a phrase $Q$ with children of type $T_{1}, \ldots, T_{k}$ and grammatical functions $G_{1}, \ldots, G_{k}$, we use the lexical probabilities

$$
P_{Q}\left(G_{i} \mid T_{i}\right)
$$

and the contextual (trigram) probabilities

$$
P_{Q}\left(T_{i} \mid T_{i-1}, T_{i-2}\right)
$$

The lexical and contextual probabilities are determined separately for each type of phrase. During annotation, the highest rated grammatical function labels $G_{i}$ are calculated using the Viterbi algorithm and assigned to the structure, i.e., we calculate

$$
\underset{G}{\operatorname{argmax}} \prod_{i=1}^{k} P_{Q}\left(T_{i} \mid T_{i-1}, T_{i-2}\right) \cdot P_{Q}\left(G_{i} \mid T_{i}\right)
$$

To keep the human annotat,or from missing errors made by the tagger, we additionally calculate the strongest competitor for each label $G_{i}$. If its probability is close to the winner (closeness is defined by a threshold on the quotient), the assignment is regarded as unreliable, and the ammotator is asked to confirm the assignment.

For evaluation, the already annotated sentences were divided into two disjoint sets, one for training ( $90 \%$ of the corpus), the other one for testing $(10 \%)$. The procedure was repeated 10 tines with different partitionings.

The tagger rates $90 \%$ of all assignments as reliable and carries them out fully automatically. Accuracy for these cases is $97 \%$. Most, errors are due to wrong identification of the subject, and different kinds of objects in sentences and VPs. Accurary of the unreliable $10 \%$ of assignments is $75 \%$, i.e., the anmotator has to alter the choice in 1 of 4 cases when asked for confirmation. Overall accuracy of the tagger is $95 \%$.

Owing to the partial automation, the average annotation efficiency improves by $25 \%$ (from around 4 minutes to 3 minutes per sentence).

\section{Conclusion}

As the annotation scheme described in this paper focusses on annotating argument structure rather than constituent trees, it differs from existing treebanks in several aspects. These differences can be illustrated by a comparison with the Penn Treebank annotation scheme. The following features of our formalism are then of particular importance:

- simpler (i.e. 'flat,') representation structures

- complete absence of empty categories

- no special mechanisms for handling discontinuous constituency

The current tagset comprises only 16 node labels and 34 function tags, yet a finely grained classification will take place in the near future.

We have argued that the selected approach is bett.er suited for producing higls quality interpreted corpora in languages exhibiting free constituent order. In general, the resulting interpreted data also are closer to semantic annotation and more nentral with respect to particular syntactic theories

As modern linguistics is also becoming more aware of the importance of larger sets of naturally occur- 

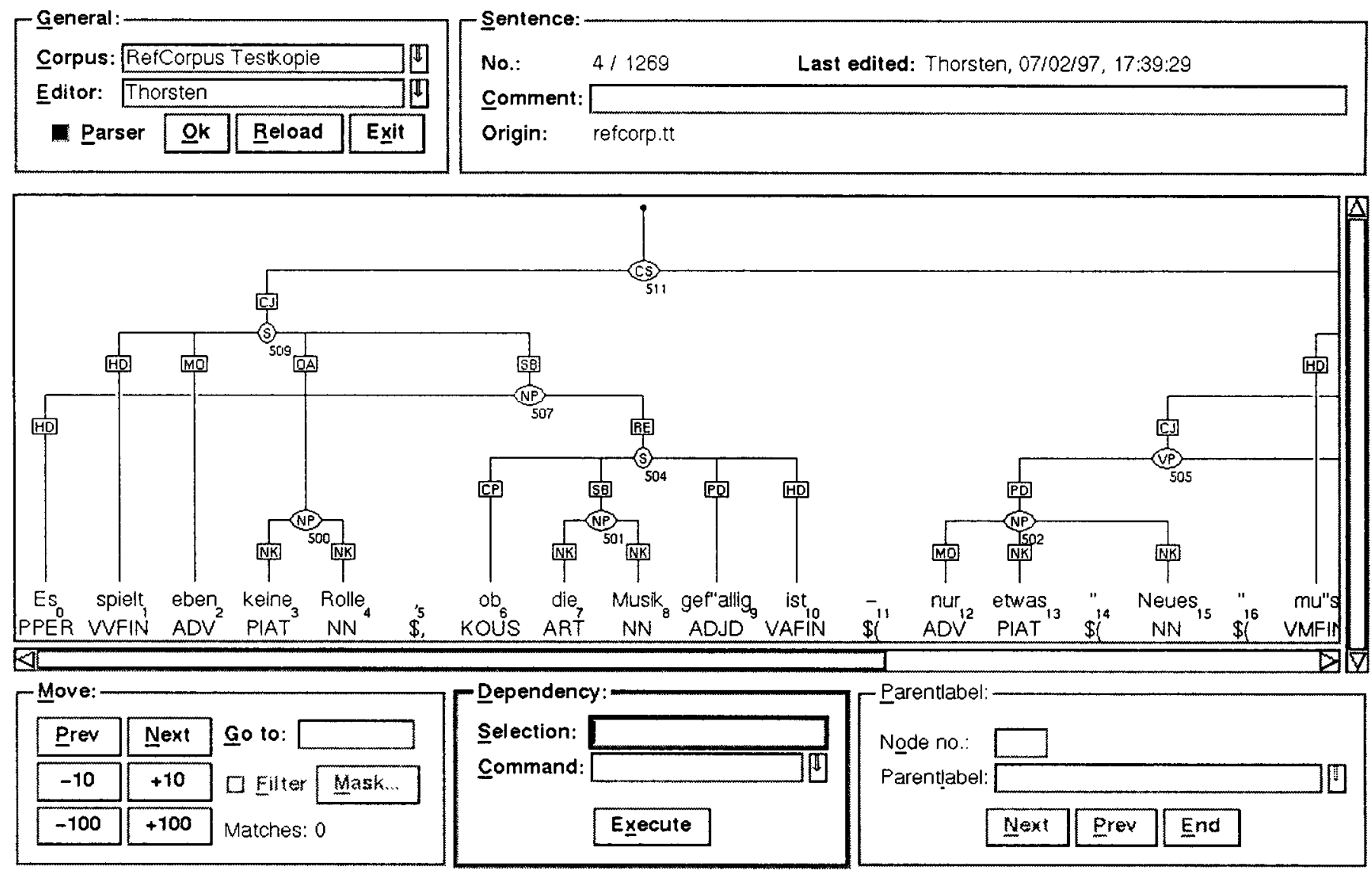

Switching to sentence no. 4... Done.

Figure 1: Screen dump of the annotation tool

ring data, interpreted corpora are a valuable resource for theoretical and descriptive linguistic research. In addition the approach provides empirical material for psycholinguistic investigation, since preferences for the choice of certain syntactic constructions, linearizations, and attachments that have been observed in online experiments of language production and comprehension can now be put in relation with the frequency of these alternatives in larger amounts of texts.

Syntactically annotated corpora of German have been missing until now. In the second phase of the project Verbmobil a treebank for 30,000 (ierman spoken sentences as vell as for the same amount of English and Japanese sentences will be created. We will closely coordinate the further development of our corpus with the annotation work in Verbmobil and with other Crerman efforts in corpus annotation.

Since the combinatorics of syutactic constructions creates a demand for very large corpora, efficiency of annotation is an important criterion for the success of the developed methodology and tools. Our annotation tool supplies efficient manipulation and immediate visualization of argunent structures. Partial automation included in the current version significantly reduces the mamal effort. Its externsion is subject to further investigations.

\section{Acknowledgements}

This work is part of the DFG Sonderforschungsbereich 378 Resourct-Adaptive Cogmitive Processes, Project (:3 Concurtent Grammar Proctssing.

We wish to thank Tania Avgustinova, Berthold Crysmann, Lars Konieczny, Stephan Oepen, Karel Oliva, Christian Weiß and two anonymous reviewers for their helpful comments on the content of this paper. We also wish to thank Robert Macintyre and Ann Taylor for valuable discussions on the Penn Treebank annotation. Special thanks go to Oliver 
Plaehn, who implemented the annotidtion tool, and to our fearless annotators Roland Hendriks, Kerstin Klöckner, Thomas Schulz, and Bernd-Paul Simon.

\section{References}

Ann Bies et al. 1995. Bracketing Guidclines for Treebank II Style Penn. Trecbank Project. Technical report, University of Pennsylvania.

Ezra Black et al. 1996. Beyond Skeleton Parsing: Producing a Comprehensive Large-Scale General-English Treebank With Full Grammatical Analysis. In The 16th International Conference on Computational Linguistics, pages 107 113, Copenhagen, Denmark.

Doug Cutting, Julian Kupiec, Jan Pedersen, and Penelope Sibun. 1992. A practical part-of-speech tagger. In Proce edings of the 3rd. Conferenct on. Applied Natural Language Proctssing (AC'L), pages 133-140.

David Dowty. 1989. Towards a minimalist theory of syntactic structure. In Tilburg Conference on Discontinuous Constituency.

Helmut Feldweg. 1995. Implementation and evaluation of a German HMM for POS disambiguation. In Proceedings of EACL-SIGDAT-95 Workshop, Dublin, Ireland.

Richard Hudson. 1984. Word Grammar. Basil Blackwell Ltd.

Fred Karlsson, Atro Voutilainen, Juha Heikkila, and Arto Anttila. 1995. Constraint Crrammar. $A$ Langnage-Independent System for Parsing Unrestricted Text. Monton de Gruyter, Berlin, New York.

Kathol, Andreas and Carl Pollard. 1995. Extraposition via Complex Domain Formation. In Proceedings of the $33^{\text {rd }}$ Anuual Meeting of the $A C L$, pages 174-180, Cambridge, MA. Association for Computational Linguistics.

Sabine Lehman et al. 1996. TSNLP - Test Suites for Natural Language Processing. In The $167 \mathrm{~h}$. International Conference on Computational Linguistics, pages $711-717$, Copenhagen, Denmark.

Mitchell Marcus et al. 1994. The Penn Treebank: Annotating Predicate Argument Structure. In Proceedings of the Human Language Technology Workshop, San Francisco. Morgan Kaufmann.

James McCawley. 1987. Some additional evidence for discontinuity. In Huck and Ojeda (eds.), Discontinuous Constituency: Syntax and Semantics, pp 185-200. New York, Academic Press.

Mike Reape. 1993. A Formal Theory of Word Order: A Cast Study in West Germanac. Ph.D. thesis, University of Ediuhurgh.
Geoffrey Sampson. 1995. Emglish for the Ciomputer. The SUSANNE Corpus.s and Analytic Schene. Clarendon Press, Oxford.

Atro Voutilainen. 1994. Designing a Parsing Grammar: University of Helsinki, Dept. of General Linguistics. Publications No. 22. 


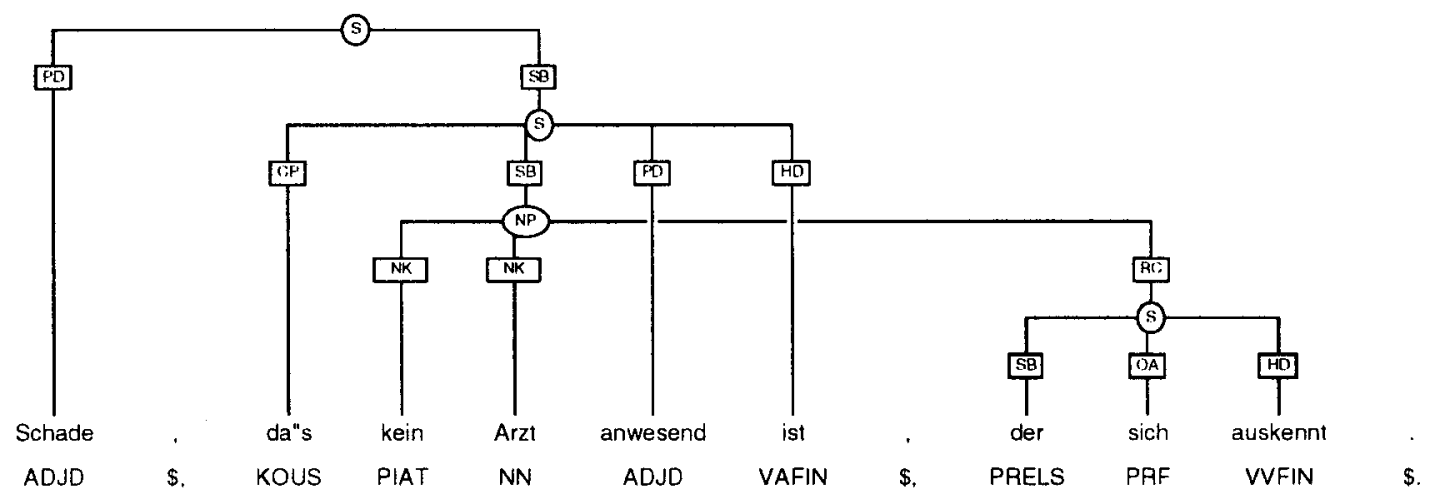

Figure 2: Headed and non-headed structures, extraposition

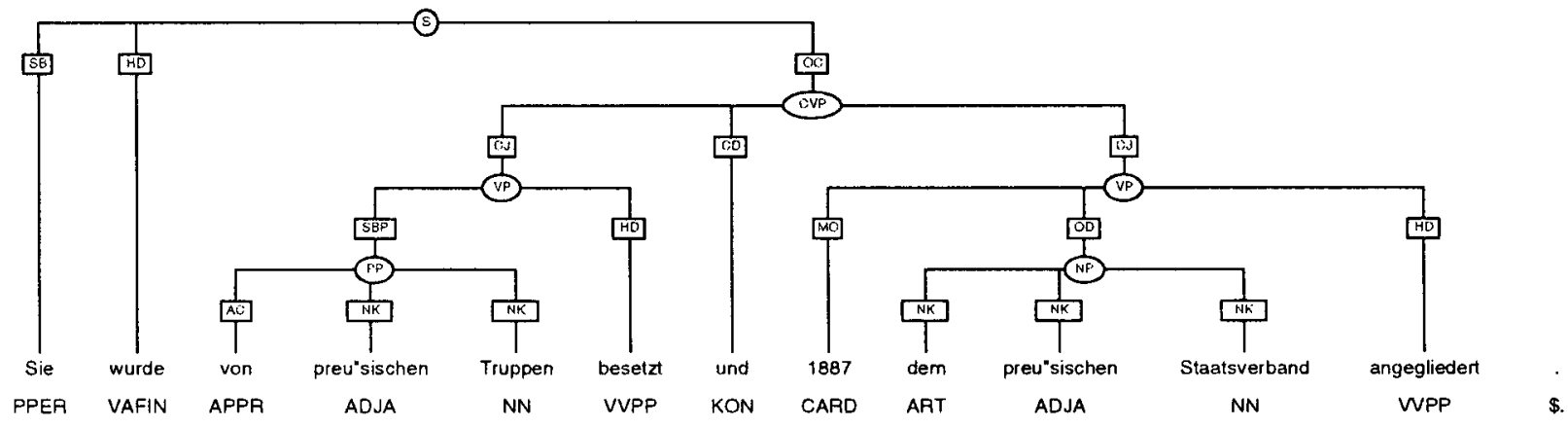

Figure 3: Coordination

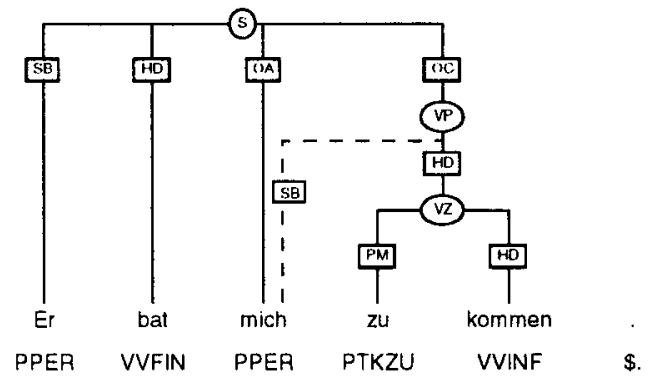

Figure 4: Ecpui construction 